\title{
Towards a conceptual approach to predetermining long-return-period avalanche run-out distances
}

\author{
Mauriae MeUnier, ${ }^{1}$ Christophe ANCEY ${ }^{2}$ \\ ${ }^{1}$ Cemagref, Domaine universitaire, 2 rue de la Papeterie, BP 76, 38402 Saint-Martin-d'Hères Cedex, France \\ E-mail:maurice.meunier@cemagref.fr \\ ${ }^{2}$ Ecole Polytechnique Fédérale de Lausanne, ENAC/ICARE/LHE, Station 18, CH-1015 Lausanne, Switerland
}

\begin{abstract}
Investigating snow avalanches using a purely statistical approach raises several issues. First, even in the heavily populated areas of the Alps, there are few data on avalanche motion or extension. Second, most of the field data are related to the point of furthest reach in the avalanche path (run-out distance or altitude). As data of this kind are tightly dependent on the avalanche path profile, it is a priori not permissible to extrapolate the cumulative distribution function fitted to these data without severe restrictions or further assumptions. Using deterministic models is also problematic, as these are not really physically based models. For instance, they do not include all the phenomena occurring in the avalanche movement, and the rheological behaviour of the snow is not known. Consequently, it is not easy to predetermine extreme-event extensions. Here, in order to overcome this problem, we propose to use a conceptual approach. First, using an avalanche-dynamics numerical model, we fitted the model parameters (friction coefficients and the volume of snow involved in the avalanches) to the field data. Then, using these parameters as random variables, we adjusted appropriate statistical distributions. The last steps involved simulating a large number of (fictitious) avalanches using the Monte Carlo approach. Thus, the cumulative distribution function of the run-out distance can be computed over a much broader range than was initially possible with the historical data. In this paper, we develop the proposed method through a complete case study, comparing two different models.
\end{abstract}

\section{INTRODUCTION}

This paper examines the use of a conceptual approach to predetermining the run-out distance of rare avalanches (i.e. those with a large period of return, basically 100 years or more). Conceptual approaches are common in hydrology, notably in the problems related to the predetermination of discharge in a given watershed from rainfall data. To our knowledge, approaches of this kind have never been fully attempted in the study of avalanche extension, although they can provide a more robust alternative to the statistical and deterministic (physical) approaches so far used for determining the run-out distance (point of furthest reach) of rare avalanches.

In the statistical approach, the basic ideas were expressed in the pioneering work of Lied and Bakkehøi (1980). These authors assumed a regional homogeneity in avalanche behaviour for a given mountain range. This allowed them to pool the data from various paths in a common database. Using regression techniques, they thus obtained a relationship between the run-out distances and various key variables of the path profile. This methodology has been applied to different mountain ranges throughout the world (see Bakkehøi and others, 1983; McClung and Lied, 1987; Fujisawa and others, 1993; Adjel, 1995) and extended to introduce the period of return as a new parameter (McClung, 2000, 2001). In Alpine countries, where the avalanche paths of the same mountain range most often exhibit no similarity in their shape, the fundamental assumption of avalanche homogeneity is questionable (Adjel, 1995).

In the deterministic approach (for a review, see Harbitz, 1999; Ancey, 2001), avalanche features are deduced by solving the equations of motion (mass and momentum equations). Deterministic models (sliding-block and depthaveraged models) introduce a friction law, reflecting the interaction between the avalanche and the path. In most models, the friction law includes two empirical frictional parameters, which have been fitted from field observations (Schaerer, 1975; Buser and Frutiger, 1980; Dent and Lang, 1980). The resulting values were proposed as default values in engineering guidelines (Swiss guidelines on the so-called Voellmy-Salm-Gubler method (Salm and others, 1990) or the U.S. Geological Survey handbook (Mears, 1992)). Though it aims primarily at providing a physical picture of avalanche motion, the deterministic approach involves too many ad hoc assumptions to be considered a true physical approach. Indeed, a number of basic physical processes (snow entrainment or release, turbulent suspension and transformation into an airborne avalanche, etc.) occurring in the avalanche course are either unknown or neglected in the avalanche-dynamics models. Furthermore, very little is known on the bulk rheological behaviour of snow, and therefore, despite a number of attempts to find physical justifications for their expressions (see, e.g., Salm, 1993), the friction laws used so far remain speculative and empirical. In this respect, it is not surprising that, in a recent benchmark of avalanche-dynamics models (see Barbolini and 
others, 2000), a significant mismatch was found in the frictional parameter values fitted from field data: this merely means that these parameters do not represent the physical properties of snow (such as snow viscosity), but, on the contrary, they reflect the interrelated influences of snow, path profile and model assumptions on the computations. Such a result is sufficiently disturbing to cast doubt on the use of avalanche-dynamics models to paths where no field data are available.

This diversity in the approaches is also encountered in hydrological sciences. As a result of more extensive practice and intense debates within a large and growing community, the ideas in hydrology on modelling are probably more precise: a clear distinction is made between statistical, physical and conceptual models (see, e.g., Betson and others, 1989; Beven, 1989; O'Connel and Todini, 1996; Bates and Campbell, 2001; Van der Kwaak and Loague, 2001). Most of the time, selecting one of these approaches depends on the level of knowledge of basic physical processes involved in the problem together with the number, quality and type of available data. Both conceptual and physical approaches represent the catchment response to a rainfall event as the result of basic processes (infiltration, storage, runoff, etc.) but in very different ways. In the physical models, the elementary processes are assumed to be known from scale-down experiments in the laboratory; the parameters introduced in the models represent physical properties that can be measured accurately and independently. On the other hand, in the conceptual models, the processes believed to be dominant in the hydrological response of a basin are idealized in the form of mathematical operators; the objective is to mimic the natural processes and not to explain them. In this case, the model parameters are purely empirical functions or values, which must be calibrated using the observable physical variables that are the object of the prediction.

It is helpful to draw a parallel between the avalanche run-out problem and the rainfall-runoff transformation. In both cases, one has input and output variables, between which one tries to find a causal/functional relationship using a physical/conceptual model. A seemingly slight difference, at first glance, does exist, however: for avalanches, the only available output information is the run-out distance, which has the drawback of being path-dependent, while in flood hydrology the selected output variable is the flow discharge, which is a truly physical variable, that is, independent of the river section at which it is measured. This difference in the type of output variable has substantial implications in developing the model since, in the latter case, it is mathematically permissible to extrapolate the probability distribution to estimate flood discharges with a long period of return, whereas, in the former case, such an extrapolation is not licit without further information. This also leads to the model parameters remaining constant for all flood events, whereas for avalanches they should vary from one event to another.

The objective pursued in this paper is to provide a proper method of extrapolating the probability distribution of run-out distances observed in a given avalanche path. Since the physical approach is questionable for the reasons given above, we have developed a conceptual model. The basic idea is to determine the dependency between the probability distributions of input and output variables for a given path. Here, this is done numerically using Monte Carlo simulations. As the input variable, we will use the starting

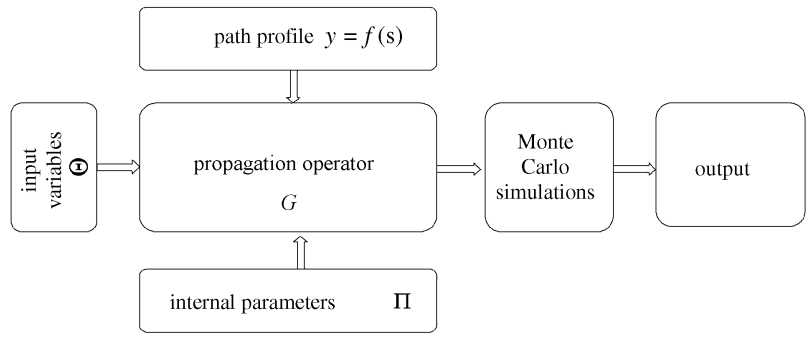

Fig. 1. Conceptual diagram of the approach.

altitude and the snow volume involved in the avalanche. An avalanche will be idealized as a sliding block and we will use a Coulomb-like model (a one-parameter model) and a Voellmy-like model (a two-parameter model) as the mathematical operators for mimicking the main features of avalanche motion. Unlike other works relying on Monte Carlo simulations (e.g. Keylock and others, 1999; Barbolini and Savi, 2001), the present method need not use questionable assumptions (such as regionalization of data) to specify the probability distributions of variables and parameters but, on the contrary, uses only the observed field data to deduce consistent probability distributions. It then introduces an important constraint: extreme avalanches are expected to have the same behaviour as observed events. If this does not seem acceptable, the method should not be used.

\section{METHODOLOGY}

If we have a time series of field data including the run-out distance $x_{\text {stop }}$, it is quite easy to deduce its empirical probability distribution. The point is that usually the time series cover a narrow period, typically a few decades. Therefore the largest period of return that can be evaluated in this way cannot exceed a few decades, while avalanche zoning requires determining the run-out distance of long-returnperiod avalanches, typically avalanches whose return period equals or exceeds 100 years. Because the empirical probability distribution $P\left(x_{\text {stop }}\right)$ is not smooth and $x_{\text {stop }}$ depends on the path profile, it is not permissible to directly extrapolate $P\left(x_{\text {stop }}\right)$ to determine the quantiles associated with low probabilities. Here we develop a model that uses available field data at best in order to properly extrapolate $P\left(x_{\text {stop }}\right)$.

Figure 1 depicts the general framework used to reach our objective. The basic idea is to assume that there is a single functional relationship $G$ between the run-out distance and other field data. This means that we need to have distinctive types of field data at our disposal to apply our method. These other field data include the snowfalls preceding the avalanche, the starting-point elevation, the released volume of snow, and so on. For the moment, we do not specify the type and number of these data but merely refer to them generically as the input variables $\Theta$. The functional relationship $G$ relates the run-out distance $x_{\text {stop }}$ of a given (fictitious or real) event to the input variables $\Theta$. Obviously there is not a oneto-one universal function linking $x_{\text {stop }}$ to $\Theta$ : indeed, it is expected that $G$ also depends on the topographical features of the path and on a set $\Pi$ of internal or structural parameters, reflecting the diversity and variability of snow consistency and avalanche motion. We express this complex relationship to take the following form: $x_{\text {stop }}=G(\Theta \mid \Pi$; path $)$. Here, in 
order to take the path influence into account, we assume that the functional $G$ is a mathematical operator resulting from the integration of a momentum equation along the path profile $y=f(s)$ (see below); in the following, we will use the short-cut notation: $x_{\text {stop }}=G(\Theta \mid \Pi)$. If the input variables $\Theta$ and/or the internal parameters $\Pi$ are random, then the run-out distance is also a random variable. If we are able to adjust the internal parameters $\Pi$ for the computed run-out distances to match the observed run-out distance, then it is possible, using Monte Carlo simulations, to create a large number of fictitious events coherent with the observations. If the run-out distance sample is large enough, we can fit an empirical probability distribution and then accurately determine the quantile related to a low occurrence probability (e.g. as low as 0.01 or 0.005 ).

Contrary to the deterministic models, the conceptual model gives results that, by definition, can be different from the measured data. To this structural error it is necessary to add the uncertainties of the data as well as of the internal parameters. Taking this into account leads to the following and more complete formulation of the model: $x_{\text {stop }}=G(\Theta \mid \Pi, \mathbf{E})$, where $\mathbf{E}$ is the uncertainty vector. The variables are taken into account through their probability distributions, within a more complete but large model. In hydrology, a more simple theoretical model is generally used, considering only an additive error $\varepsilon$, leading to $x_{\text {stop }}=G(\Theta \mid \Pi)+\varepsilon$. However, in hydrology, internal parameters of conceptual models are constant for each event, which is not the case for avalanche models. Further research is needed to determine whether the use of a simply additive error is consistent in our field. Yet we already know that the indirect linkage between the probability distributions of the internal parameters and the distribution of $x_{\text {stop }}$ gives results similar to the direct inversion method used in the first step of the methodology below (Ancey and others, 2003). So, we will develop the entire methodology using this direct method and will not use the complicated error formulation in this first attempt at the conceptual approach.

After outlining the general principles, we will explain how the method can be applied in practice. The method can be broken down into four steps:

The first step selects the input variables among all the available field data and fits $\Pi$. Usually the avalanche database has various types of information available (snowfall, volume, etc.), but not all the information can be used. For instance, redundant data must be set aside. Similarly, data whose time series is not complete or not consistent with other time series cannot be retained as input variables of the model. Next, the different values of $\Pi$ are adjusted for each documented event. Different strategies can be used to solve this inverse problem. In hydrology, a current practice is to use Bayesian inference to deduce $p(\Pi)$ from $p(\Theta)$ and $p\left(x_{\text {stop }}\right)$, where $p(X)$ is the probability density function (PDF) of the random variable $X$. Deterministic methods can be used equally for this purpose (see, e.g., Ancey and others, 2003). Here we directly compute $\Pi$ for each event and then we adjust a probability distribution to the resulting sample of values $\Pi$. All these methods have a common impediment in that we have a single type of output variable, whereas the dimension of $\Pi$ may be larger than unity. In the case where $\Pi=\left\{\pi_{1} \cdots \pi_{n}\right\}$ with $n \geq 2$, only one parameter $\pi_{i}$ can be determined, provided that other parameters $\pi_{j}$ with $j \neq i$ are known; therefore, we have: $\pi_{i}=G^{-1}\left(x_{\text {stop }}, \Theta \mid \pi_{j}\right)$. In practice, using propagation operators involving a large number of internal parameters leads to substantial complications.

In the second step, attention is paid to obtaining the PDFs of the input variables $p(\Theta)$ and of the internal parameters $p(\Pi)$ (if the latter has not been determined in the first step). Since the data are available, this merely means that we try to adjust usual probability distributions (Gumbel, Pearson, normal, etc.) from the selected field data and the internal parameters.

In the third step, Monte Carlo simulations are performed. For each fictitious event, random realizations of $\Pi$ and $\Theta$ are generated from their respective probability density function.

In the fourth step, the run-out distance is computed by applying the propagation operator to these random vectors and then it is stored. Avery large sample of $x_{\text {stop }}$ can be obtained in this way. We then deduce the empirical PDF of $x_{\text {stop }}$. This function must approximately match the empirical distribution adjusted from the recorded distances but, since it results from a much larger sample, it extends over a wider range of probabilities. This allows us to accurately compute the quantile associated with a low non-exceedance probability. For instance, the runout distance whose period of return is 500 years (corresponding to a non-exceedance probability of 0.002) can be determined by generating 1000 years or more.

\section{Propagation operator}

In this paper, we examine a propagation operator involving one or two internal parameters. Here an avalanche is idealized as a solid mass sliding along a curvilinear path and experiencing a frictional force $F$, possibly depending on $\theta$ and/ or $u$ ( $\theta$ is the local slope, $u$ is the local velocity). In a first approximation, we assume that the structure of this frictional force is identical whatever the path and the avalanche; only its parameters can vary from one event to another, but they remain constant during the entire course of an avalanche. The general expression of the momentum equation is written ( $m$ is the avalanche mass, $t$ is time):

$$
\frac{\mathrm{d} u}{\mathrm{~d} t}=g \sin \theta-\frac{F(\theta, u)}{m} .
$$

As initial conditions, we use $u\left(x_{\text {start }}\right)=0$, where $x_{\text {start }}$ is the starting-point abscissa. The momentum equation is integrated along the path profile $y=f(s)$, where $y$ denotes the elevation and $s$ the abscissa along a horizontal axis; $x$ is a curvilinear abscissa taken from an arbitrary origin on the path profile: $x=\int_{0}^{s}\left(1+f^{\prime 2}(\sigma)\right)^{1 / 2} \mathrm{~d} \sigma$. After integrating the equation numerically, we look for the position of the stopping point at which the avalanche velocity vanishes. We refer to this point as the run-out point or distance $x_{\text {stop. }}$. In Equation (1), two expressions of the frictional force have been tested:

One-parameter expression (Coulomb-like model): the force $F$ assumed to be slope-dependent $F=\mu m g \cos \theta$, where $\Pi=\{\mu\}$ is the internal parameter.

Two-parameter expression (Voellmy-like model): the force $F$ is split into a slope-dependent term and a velocity-dependent term: $F=\mu m g \cos \theta+\kappa u^{2}$, where 


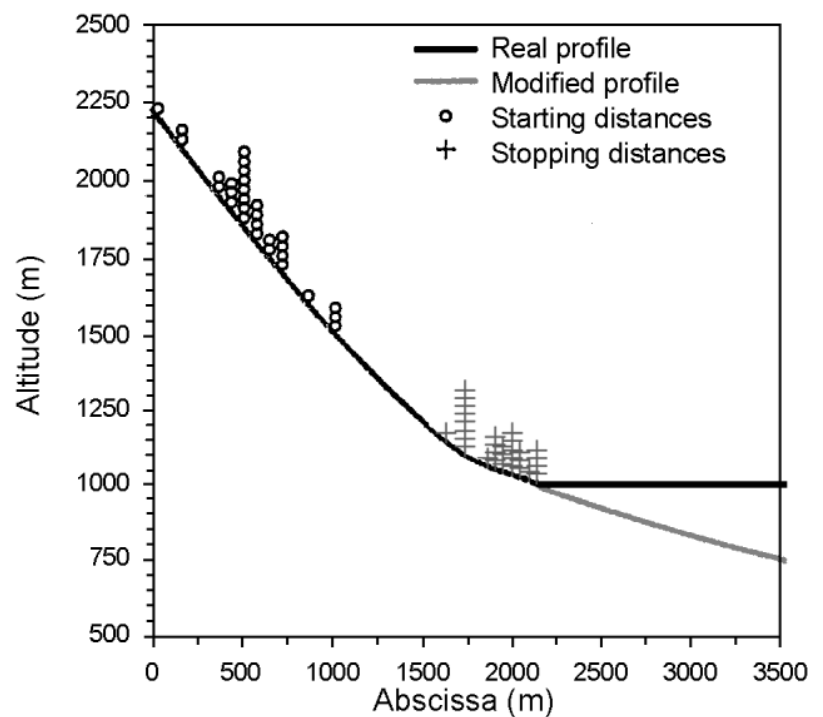

Fig. 2. Slope profile of the Entremène avalanche path.

$\Pi=\{\mu, \kappa\}$ are the two internal parameters. The former contribution makes it possible to control the avalanche extent while the latter mainly influences the maximum velocity that the avalanche can reach. Moreover, it has often been recognized that the avalanche mass or volume often influences the force: the larger volume $V$ is, the lower its bulk friction is. Thus, parameter $\kappa$ must be a function of the avalanche volume. For convenience, here we assume that this dependency can be written in the following form: $\kappa=g /(\xi H)$, where $\xi$ is a friction coefficient and $H \propto \sqrt{V}$ is a typical length assumed to give an estimate of the mean flow depth of the avalanche. Using heuristic arguments presented in the Appendix, we will use the following ad hoc relationship between $H$ and $V: H=2.5+5 \times 10^{-3} V^{1 / 2}$.

\section{APPLICATION}

\section{Selected avalanche path and data}

For the case study, we have selected the Entremène avalanche path, which is situated on the left side of the Arve river in the Chamonix valley, France. This choice was motivated by two different considerations: first, avalanche activity is substantial and regular; thus we have a long time series of avalanche data on this path (approximately 100 years); second, the upper part of the profile is sufficiently smooth and open for the avalanche dynamics to be simple and similar for each event. In the lower part of the profile, there is a sharp transition in the path slope since, at an elevation of approximately $1000 \mathrm{~m}$, the path is very close to horizontal (Fig. 2). This transition significantly affects avalanche motion since several avalanches stopped in the transition zone. In order to test the influence of the profile on the distribution of extreme run-out distances, we will also consider a modified path profile, for which the slope discontinuity was smoothed. Both profiles are depicted in Figure 2.

With the conceptual approach, the quality of the results is largely dependent on the quality of the data; it is necessary to have the best and the longest dataset possible, containing exactly the variables that are needed by the model: in our case, because we want to use the Voellmy model, it is neces-
Table 1. Avalanche data on the Entremène path and $\mu$ values for the Coulomb-like model

\begin{tabular}{|c|c|c|c|c|}
\hline \multirow[t]{2}{*}{ Date } & \multirow{2}{*}{$\begin{array}{l}Z_{\text {start }} \\
\mathrm{m}\end{array}$} & \multirow{2}{*}{$\begin{array}{l}Z_{\text {stop }} \\
\mathrm{m}\end{array}$} & \multirow{2}{*}{$\begin{array}{l}\text { Volume } \\
\mathrm{m}^{3}\end{array}$} & \multirow[t]{2}{*}{$\begin{array}{c}\text { Coulomb-like model } \\
\mu_{\text {Coul }} \text { values }\end{array}$} \\
\hline & & & & \\
\hline 19 Mar. 1905 & 2200 & 1050 & 3000 & 0.6167 \\
\hline 14 Jan. 1909 & 2100 & 1050 & 11520 & 0.6071 \\
\hline 20 Jan. 1910 & 1850 & 1010 & 478800 & 0.5319 \\
\hline 18 Nov. 1910 & 1800 & 1100 & 67200 & 0.6167 \\
\hline 8 Jan. 1912 & 1800 & 1030 & 32400 & 0.5468 \\
\hline 23 Jan. 1913 & 1850 & 1060 & 24000 & 0.5918 \\
\hline 26-27 Mar. 1914 & 2100 & 1020 & 72960 & 0.5751 \\
\hline 19 Feb. 1916 & 1950 & 1020 & 252000 & 0.5567 \\
\hline 28 Mar. 1919 & 1900 & 1040 & 82500 & 0.5727 \\
\hline 24-25 Dec. 1919 & 1850 & 1020 & 288000 & 0.5427 \\
\hline 9 Jan. 1922 & 1900 & 1040 & 136800 & 0.5727 \\
\hline 2 Mar. 1923 & 1850 & 1040 & 140000 & 0.5661 \\
\hline 23 Dec. 1923 & 1900 & 1030 & 150000 & 0.5610 \\
\hline 27 Dec. 1925 & 1850 & 1030 & 86400 & 0.5542 \\
\hline 14 Feb. 1928 & 1750 & 1100 & 192500 & 0.6111 \\
\hline 31 Jan. 1929 & 1950 & 1100 & 15750 & 0.6316 \\
\hline Apr. 1935 & 1600 & 1100 & 18000 & 0.5909 \\
\hline 12 Jan. 1938 & 1850 & 1100 & 37500 & 0.6220 \\
\hline 30 Jan. 1938 & 1750 & 1100 & & \\
\hline 9 Mar. 1939 & 1700 & 1050 & 7500 & 0.5568 \\
\hline 2 Jan. 1943 & 1700 & 1050 & & \\
\hline 8 Dec. 1944 & 1700 & 1030 & 144000 & 0.5301 \\
\hline 11-12 Jan. 1947 & 1800 & 1100 & 105000 & 0.6167 \\
\hline 9-10 Feb. 1950 & 1800 & 1030 & 96000 & 0.5468 \\
\hline 20 Jan. 1951 & 1850 & 1000 & 90000 & 0.5218 \\
\hline 28 Feb. 1952 & 1850 & 1000 & 2700 & 0.5218 \\
\hline 24 Feb. 1957 & 1500 & 1150 & 48000 & 0.5925 \\
\hline 14 Mar. 1958 & 1500 & 1000 & 48000 & 0.4503 \\
\hline 29 Mar. 1962 & 1500 & 1100 & & \\
\hline 3 Feb. 1970 & 1700 & 1000 & 108000 & 0.4957 \\
\hline
\end{tabular}

sary to include the heights of the avalanche events, deduced from the volume deposits, in order to restrict the sample size to the period where the deposit volumes have been estimated.

The avalanche database includes 59 events since 1905 . Not all of these events have been recorded; notably, the avalanches stopping in the upper part of the site were not taken into account. For the period 1905-70, the observed data were the starting and stopping elevations and the deposit volume; in Table 1, 30 events are presented but, for three of them, no information on the deposit volume is available. In Figure 2, we have reported the different starting and stopping points of the 27 avalanche events for the period 190570. Since 1971, the deposit volume has no longer been estimated. For the recent period (1970-2002), 29 events have been recorded and will not be used here. This is a pity since we will use only half of the available events from the entire recorded period, and it is obvious that large samples give more accurate results. However, it does not seem possible to easily generate the missing volume data of the recent period in order to increase the size of samples. The Monte Carlo simulation may provide a solution. Furthermore, the number of events in the period used is simlar to that in the shorter recent period. There is seemingly a problem of stationarity, which should be studied for an engineering application, but this will not be dealt with in this methodological paper. Likewise, the influence of the sample size on the result accuracy will not be studied.

Uncertainty on the run-out elevation varies with time. 


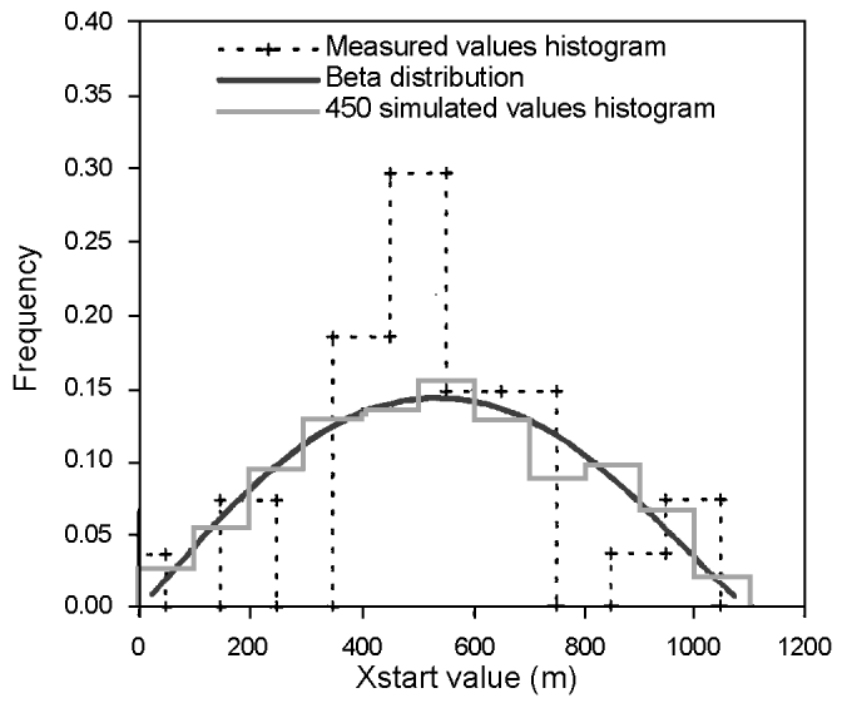

Fig. 3. Statistical distribution of the avalanche starting distances.

At the beginning of the 20th century, it probably exceeded $\pm 100 \mathrm{~m}$, while nowadays it is expected to be much lower $( \pm 25 \mathrm{~m})$. If we use distance rather than elevation to describe avalanche run-out, then uncertainty on the stopping-point position is amplified for the path parts where the slope is low, typically here in the nearly horizontal part of the profile. Uncertainty on the volumes is very large: on this path, it has been estimated as high as 50\% according to the deposit dimension measurements. We must also add the uncertainty due to the shape of the deposit. This large uncertainty may have consequences for the methodology results. A specific study could improve this point, but this present study will investigate only the confidence limits which are generated by the method itself.

In conclusion, the analysis of the available dataset is fundamental in the conceptual approach; consequently, important statistical questions arise, replacing the deterministic questions occurring in previous studies.

\section{Procedure with the Coulomb-like model and results}

For the first step, we chose the input and output variables: the starting and run-out distances $x_{\text {start }}$ and $x_{\text {stop }}$. By fitting the model, we obtained the sample of friction parameters (see Table 1).

For the second step, we first fitted the sample of $x_{\text {start }}$ values. Since $x_{\text {start }}$ is bounded, it is very convenient to use the beta distribution as the probability distribution. Figure 3 shows that the frequencies of the middle classes in the experimental values are higher than the fitted distribution. The experimental histogram is very irregular (e.g. for some classes, empirical frequency is zero). We fitted a Gumbel distribution to the $\mu$ sample, as shown in Figure 4 . The Gumbel distribution was used in a first approximation; other possibilities will be explored below, such as the normal distribution, which is included in Figure 4 and whose adequacy seems better than the Gumbel distribution.

In the third step, we created large samples of $x_{\text {start }}$ and $\mu$ values using a random-number generation routine. As the experimental sample contains 27 events for 60 years, the simulated sample for 1000 years should contain 450 events. Figures 3 and 4 show the simulated values for $x_{\text {start }}$ and $\mu$,

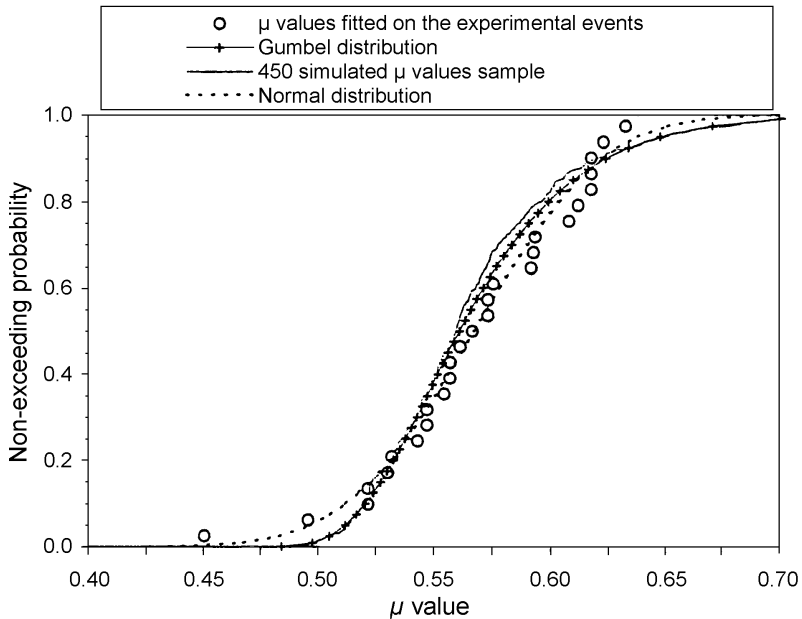

Fig. 4. Probability distribution of the $\mu$ values (Coulomb-like model).

respectively; these samples are very close to the theoretical distributions.

In the last step, we used these two samples to generate 450 fictitious avalanches. The run-out distance of each event was stored. As shown in Figure 5, there is little difference between the empirical probability distribution of the simulated run-out distances computed using the Coulomblike model and the Voellmy-like model respectively (see below). The simulated run-out distances match the field data well, except for the last four identical points (run-out distance at $2100 \mathrm{~m}$ ). At this critical point, the path profile is nearly horizontal (river bed) and, as stated previously, uncertainty on the stopping-point position is high. For an operational study, it would be necessary to evaluate the genuine run-out distances with an historical analysis. Fitting the distributions will be more convenient for these four experimental points. However, in the conceptual approach all the experimental points are considered in the evaluation of the fitness quality and not only the extreme points. Thus, we conclude that on the whole, the model correctly describes the past avalanche activity in the Entremène path.

The 500 year run-out distance obtained is $2269 \mathrm{~m}$. This result is unique since the model has only one parameter and we used a single distribution for $x_{\text {start }}$ and $\mu$. We will see below what happens with different distributions.

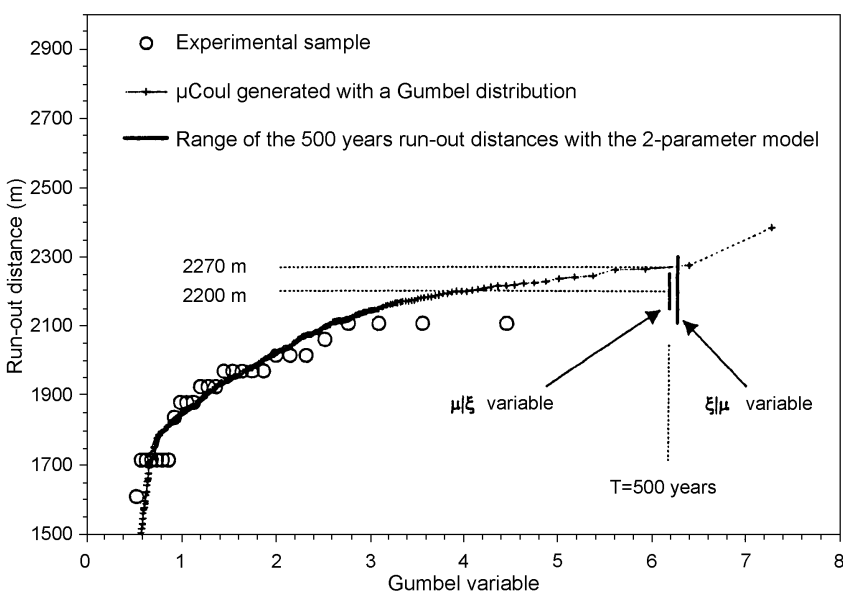

Fig. 5. Run-out distance statistical distributions obtained with the Coulomb-like model and the Entremène real profile. 


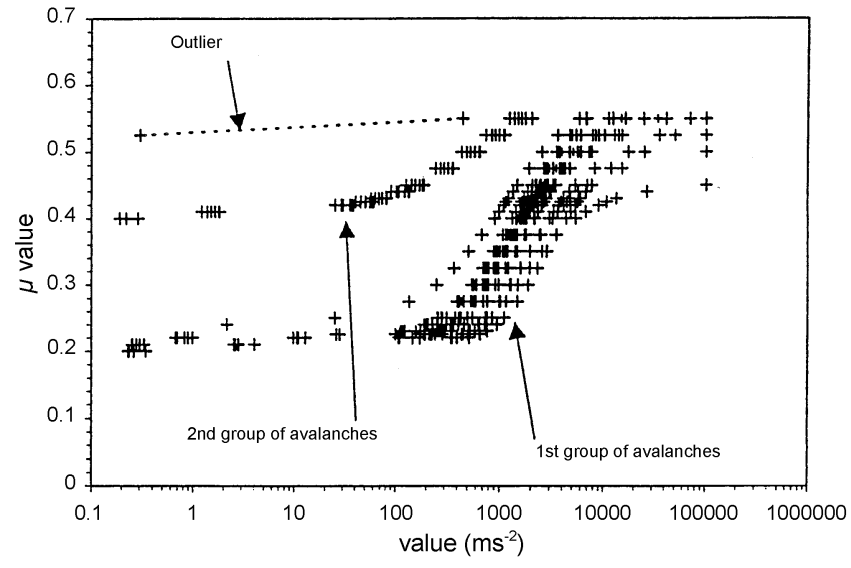

Fig. 6. Computed $\xi$ values with fixed $\mu$.

\section{Procedure with the Voellmy-like model and results}

In the first step, we computed the input and output variables together with the friction coefficients. The Voellmy-like model involves two input variables: the initial condition $x_{\text {start }}$ value and the avalanche depth $H$. We verified that they are not correlated. The model has two internal parameters $(\mu, \xi)$. Since we have only one output variable, the value of one of them must be kept constant for the inverse problem to be solved. In the following, we will use the notation $(\xi \mid \mu)$ when it is considered that the parameter $\xi$ is free while the other parameter $\mu$ is held constant for all the events occurring in the path. Figure 6 provides the values of the friction parameter $\xi$ that were deduced from the field data when the value of the other friction parameter $\mu$ is held constant for all events. A very similar figure (not reported here) was obtained when computing the $\mu$ values for a varying $\xi$ parameter. In either case, the figure exhibits outliers from the same events, which we will ignore later on. It is also worth noting that:

the curve $\mu(\xi)$ is horizontal for low and large values of $\xi$. This gives a first argument for reducing the range of $\xi$

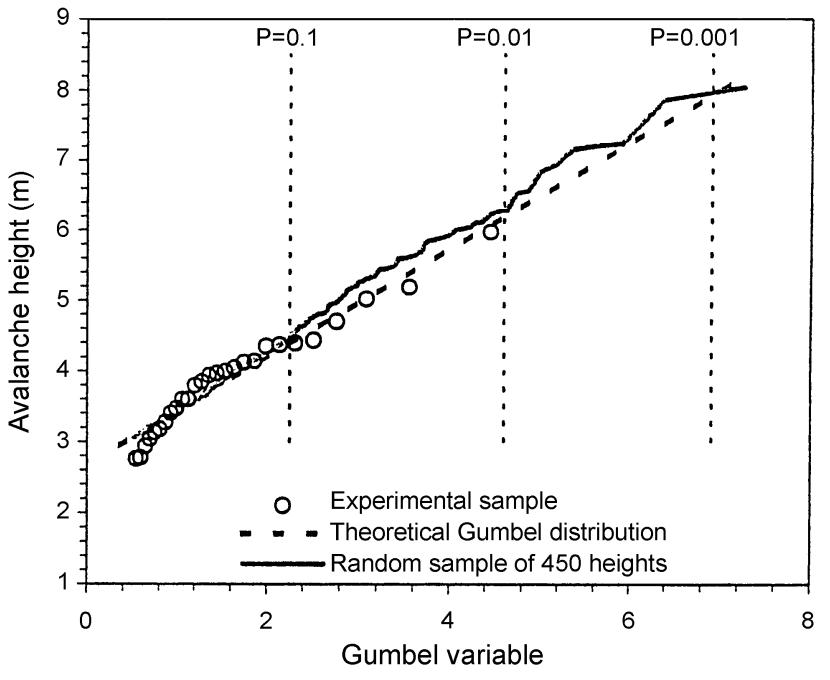

Fig. 7. Statistical distribution of the avalanche heights.

values: $\mu$ being fairly constant for the lowest and highest $\xi$ values, we can consider that $\xi$ ranges from 500 to $10000 \mathrm{~m} \mathrm{~s}^{-2}$ without loss of generality. Other arguments below can be used to narrow this range further.

the data scattering defines two event families (denoted as the first and second group in Figure 6): for a given $\xi$, there is no continuum in the $\mu$ values, but, on the other hand, two narrow ranges of possible values can be observed. This clear separation in the fitted $\mu$ values has also been observed on other paths when the Voellmy model is used (Ancey and others, 2003), and no convincing explanation has been found. On the Entremène path, the separation of the $\mu$ values corresponds to a separation of the stopping altitudes, below and above 1070$1100 \mathrm{~m}$ a.s.l.

In the second step, we first verified that the input variables were independent and necessary. Most authors who used a sliding-block model (or, in fact, any avalanchedynamics model) did not consider the starting-point

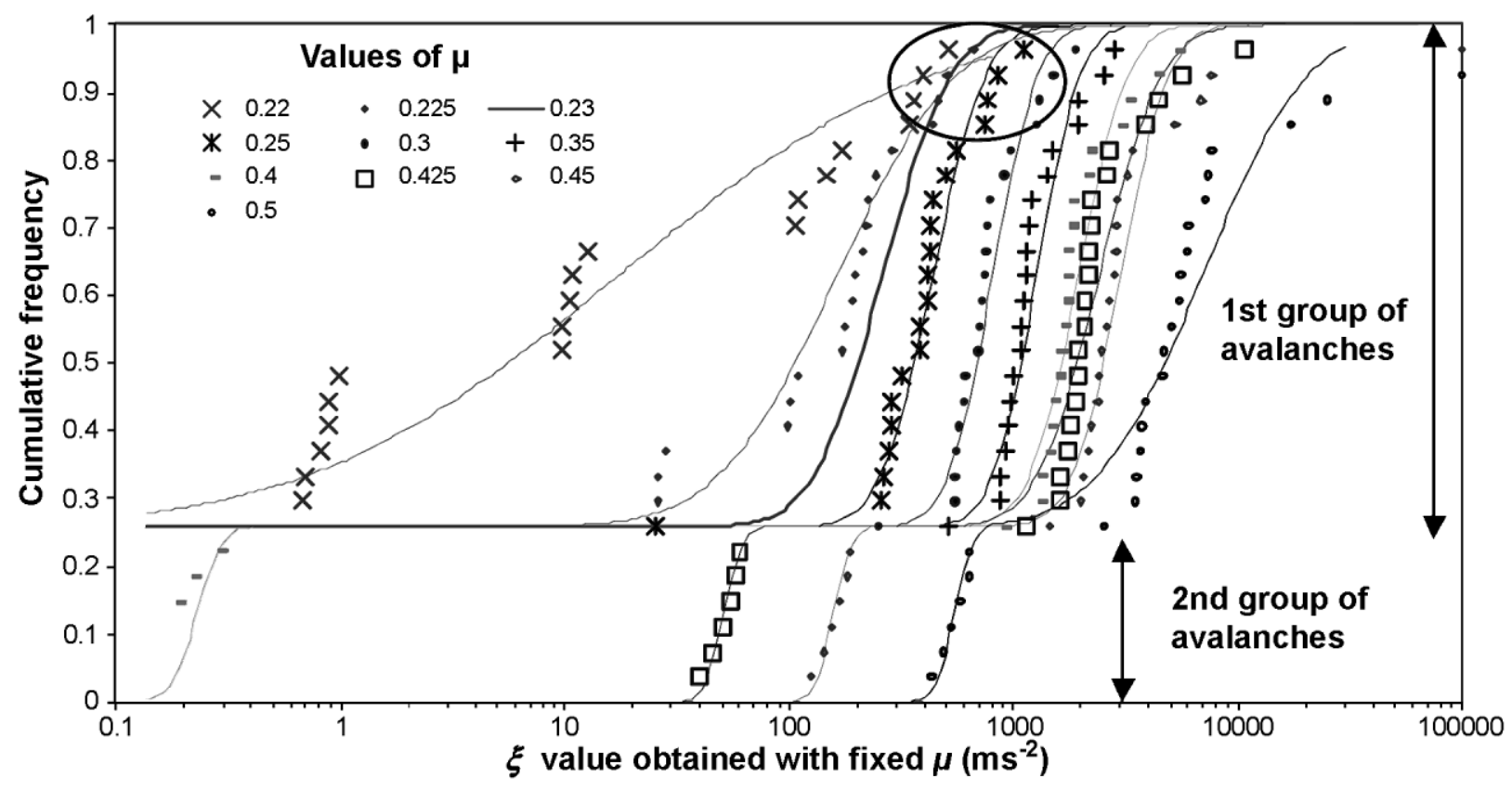

Fig. 8. One variable conditional probability distribution of $(\xi \mid \mu)$ with fixed $\mu$ (Voellmy-like model). 


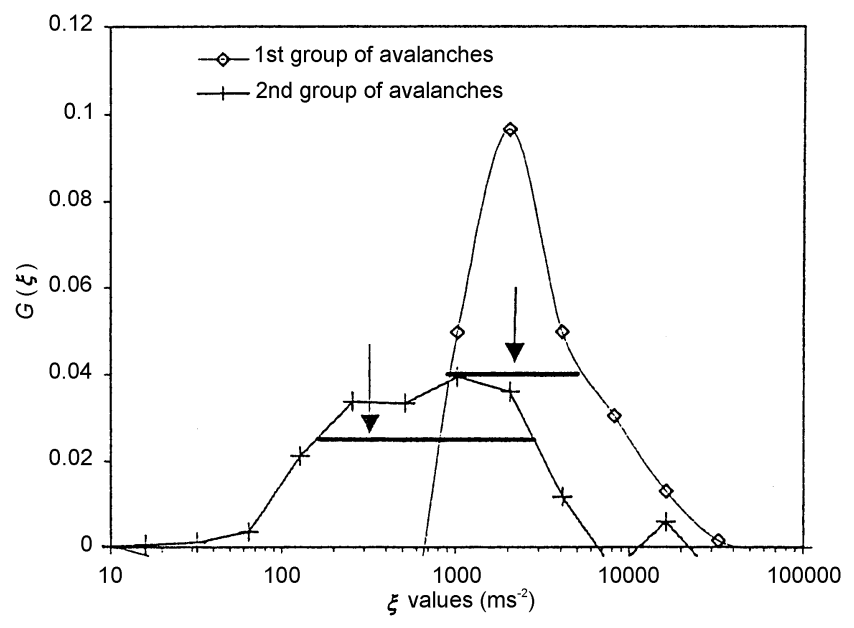

Fig. 9. Determination of the reduced range of $\mu$ for the Vollmy-like model (the arrows indicate the limit of validity of the criterion).

position as a reliable dynamics parameter since the initial conditions would affect neither the steady-state solution nor the run-out distance. We examined this point more closely: for all the experimental events, we computed the runout distances with several fixed values of $\xi$, the value of $\mu$ (fitted to the observed events) and a fixed $x_{\text {start }}$ value. The mean difference between the recorded and simulated runout distances was found to vary as a function of the $\xi$ value. For $\xi$ values as high as $1000 \mathrm{~m} \mathrm{~s}^{-2}$, the mean difference is close to zero and, in this case, the starting-point position need not be considered as an input parameter. However, for $\xi>1000 \mathrm{~m} \mathrm{~s}^{-2}$, this no longer holds. The mean difference increases substantially with $\xi$ and can exceed $40 \mathrm{~m}$ for $\xi>5000 \mathrm{~m} \mathrm{~s}^{-2}$. In short, surprisingly enough and contrary to common belief, it is necessary to include the input variable $x_{\text {start }}$ in the computations since the run-out distance may depend on $x_{\text {start }}$ for sufficiently large values of $\xi$. A beta distribution was used to fit the $x_{\text {start }}$ values. For the avalanche depth $H$, a Gumbel distribution fits the data well (to the naked eye), as shown in Figure 7.

Determining the statistical distributions on the $(\xi \mid \mu)$ and $(\mu \mid \xi)$ samples turns out to be intricate since it is not permissible to use a one-peak distribution fitted to the data. The simplest approximation involves taking the sum of the two probability distributions fitted on each group. In this way, we obtain the one-variable conditional distribution of each parameter. In a previous investigation, we found that a probability distribution made up of two beta distributions fitted the $(\mu \mid \xi)$ values well (Meunier and others, 2001). However, since in the following we are also interested in determining the $(\xi \mid \mu)$ distribution, we find it simpler to fit a statistical model resulting from superposing two normal distributions on the $(\mu \mid \xi)$ and $(\ln \xi \mid \mu)$ values; here we use $(\ln \xi \mid \mu)$ rather than $(\xi \mid \mu)$ because the range of possible $\xi$ values covers several orders of magnitude. As shown in Figure 6 for $\xi$, we have explored the entire range of possible values for the two parameters, even though they may have no physical meaning for the practitioner. Figure 8 shows the cumulative distribution functions fitted to the $(\ln \xi \mid \mu)$ values. These figures call for several remarks:

The existence of two event groups is fully conditioned by the $\mu$ value: for $\mu<0.4$, the second group does not exist
Table 2. Range of practical interest for the friction parameters (Vollmy-like model)

\begin{tabular}{lcc}
\hline $\begin{array}{l}\text { Group of } \\
\text { avalanches }\end{array}$ & $\begin{array}{c}\text { Practical range for } \mu \text { in order to } \\
\text { have } \xi \text { as a random variable }\end{array}$ & $\begin{array}{c}\text { Practical field for } \xi \text { in order to } \\
\text { have } \mu \text { as a random variable } \\
\mathrm{m} \mathrm{s}^{-2}\end{array}$ \\
\hline First & $0.23-0.46$ & $900-5000$ \\
Second & $0.43-0.55$ & $165-2800$ \\
\hline
\end{tabular}

because, in this case, the computed run-out distances $x_{\text {stop }}(\xi \mid \mu)$ never match the field data.

For extreme $\mu$ values (i.e. when $\mu \leq 0.225$ or $\mu \geq 0.5$ ), agreement between empirical and computed frequencies is poor: the empirical distribution is step-shaped while the fitted distribution tends to smooth abrupt variations in the $\xi$ value.

For these extreme $\mu$ values, the fitted sum of two normal distributions is no longer parallel to the others, which implies that the curves intersect. This point is disturbing: if we keep them, inconsistent values will be generated when we apply the Monte Carlo method. Obviously this undesirable behaviour originates from the fact that the curve $\mu(\xi)$ flattens out when $\xi \ll 10 \mathrm{~m} \mathrm{~s}^{-2}$ : in this case, low variations in the $\mu$ value induce large variations in the $\xi$ value.

This motivated us to reduce the $\mu$ range by removing values that provide the non-parallel curves in Figure 8. Since we use a sum of two normal distributions, this also means that we discard the $\mu$ values as soon as both the mean $\bar{\xi}$ and the standard deviation $\sigma_{\xi}$ of the $(\ln \xi \mid \mu)$ sample differ significantly from a set of selected values. Translated into a mathematical expression, this condition can be expressed as follows. If the condition:

$$
F(\mu)=\left|\frac{\mathrm{d} \bar{\xi}}{\mathrm{d} \mu}+\frac{\mathrm{d} \sigma_{\xi}}{\mathrm{d} \mu}\right| \leq k
$$

is not fulfilled, in which $k=20$ for the first avalanche group and $k=55$ for the second avalanche group, then the $\mu$ value must be put aside. Using this criterion, we found that $\mu$ must range from 0.23 to 0.46 for the first group while $\mu$ must fall within the range $0.43-0.55$ for avalanches belonging to the second group. The same exercise was done by inverting the role of $\mu$ and $\xi$. The same analysis as previously gives the same conclusions and leads to a similar criterion (see Fig. 9):

$$
G(\xi)=\frac{\mathrm{d} \bar{\mu}}{\mathrm{d} \ln \xi}-6\left|\frac{\mathrm{d} \sigma_{\mu}}{\mathrm{d} \ln \xi}\right| \geq k^{\prime},
$$

where $k^{\prime}=0.04$ for the first group and $k^{\prime}=0.025$ for the second. Note that here, because the derivatives of the mean and the standard deviation do not have the same order of magnitude, we multiply the derivative of the standard deviation by 6 in order to give the same weight to the two derivatives. In the end, we find that the $\xi$ range is 900$5000 \mathrm{~m} \mathrm{~s}^{-2}$ for the first group of avalanches and 165$2800 \mathrm{~m} \mathrm{~s}^{-2}$ for the second group. The resulting ranges of the friction parameters are shown in Table 2.

The third step is much easier: for each input variable, a sample of 450 values was randomly generated from its adjusted empirical probability distribution (see Fig. 2 for $x_{\text {start }}$ and see Fig. 7 for $H$ ). Similarly, 450 values of the 


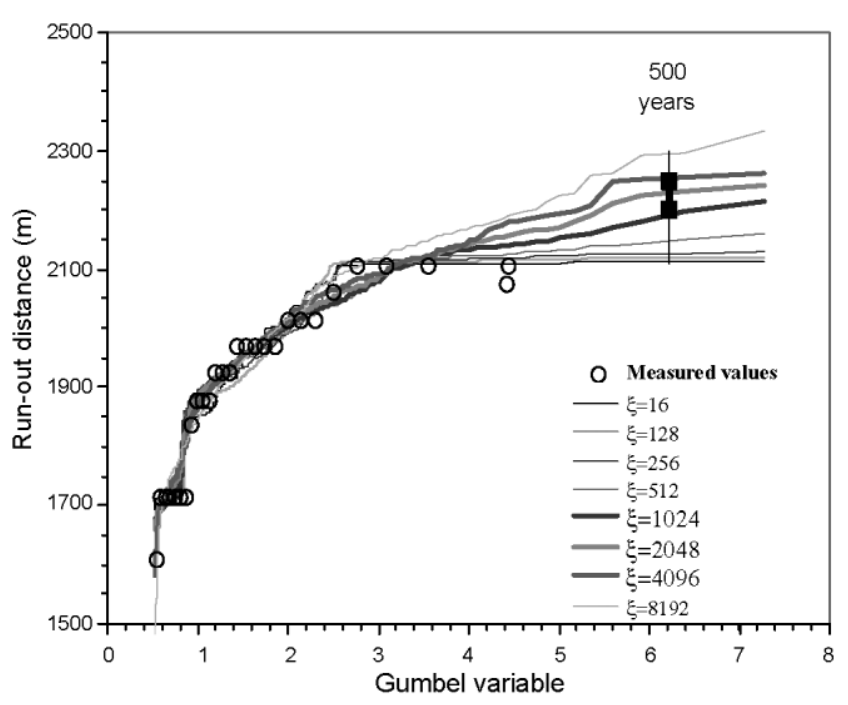

Fig. 10. Run-out distance statistical distributions with the $\mu$ samples, different fixed values of $\xi\left(\right.$ in $\mathrm{m} \mathrm{s}^{-2}$ ), and the real profile (Vollmy-like model).

internal parameters were created from the different conditional distribution of $(\ln \xi \mid \mu)$ or $(\mu \mid \xi)$.

In the fourth step, the run-out distances were computed and stored. Figure 10 reports the statistical distributions of the run-out distances obtained when using the $(\mu \mid \xi)$ samples. Let us examine what happens when we consider extreme events that could occur in the future or that could have occurred in the distant past. A key point is that the bundle of curves diverges for run-out distances in excess of the critical point $(2100 \mathrm{~m})$. Data scattering is pronounced (200 $\mathrm{m}$ for the 500 year return-period run-out distance) when the entire range of $\xi$ is considered. In contrast, when we focused our attention on the limited range of $\xi$ determined previously, we found that the range of 500 year runout distance is narrow: approximately $50 \mathrm{~m}$.

We proceeded similarly with the $(\xi \mid \mu)$ sample. The results are reported in Figure 11. The range for 500 year run-out distance was similar for the entire or limited ranges of $\mu$ (120 m vs $90 \mathrm{~m})$. However, the mean values for the practical range were somewhat different: larger for the $(\mu \mid \xi)$ simulations $(2228 \mathrm{~m})$ than for the $(\xi \mid \mu)$ simulations (2206 m). Yet the difference was only $22 \mathrm{~m}$ (see Table 3 ).

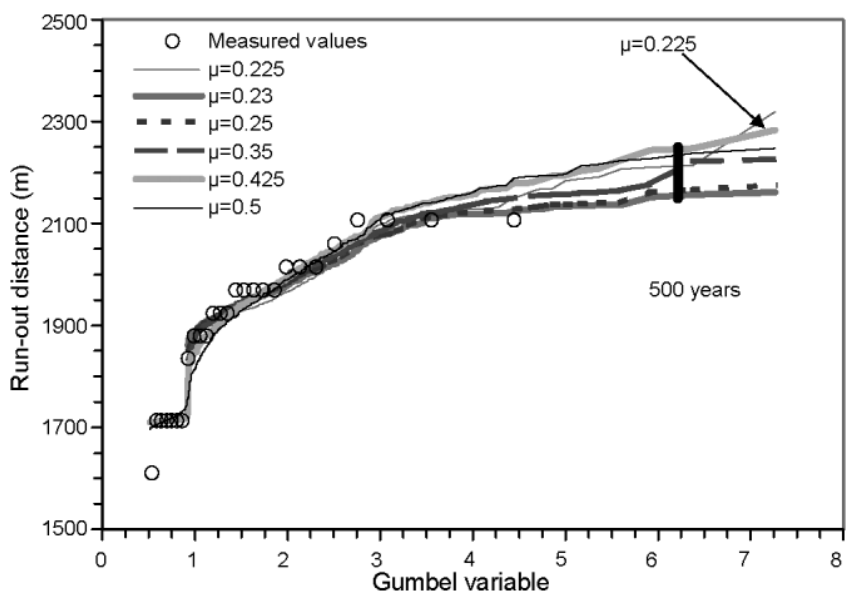

Fig. 11. Run-out distance statistical distributions with the $\xi$ samples (in $\mathrm{m} \mathrm{s}^{-2}$ ), different fixed values of $\mu$, and the real profile (Vollmy-like model).

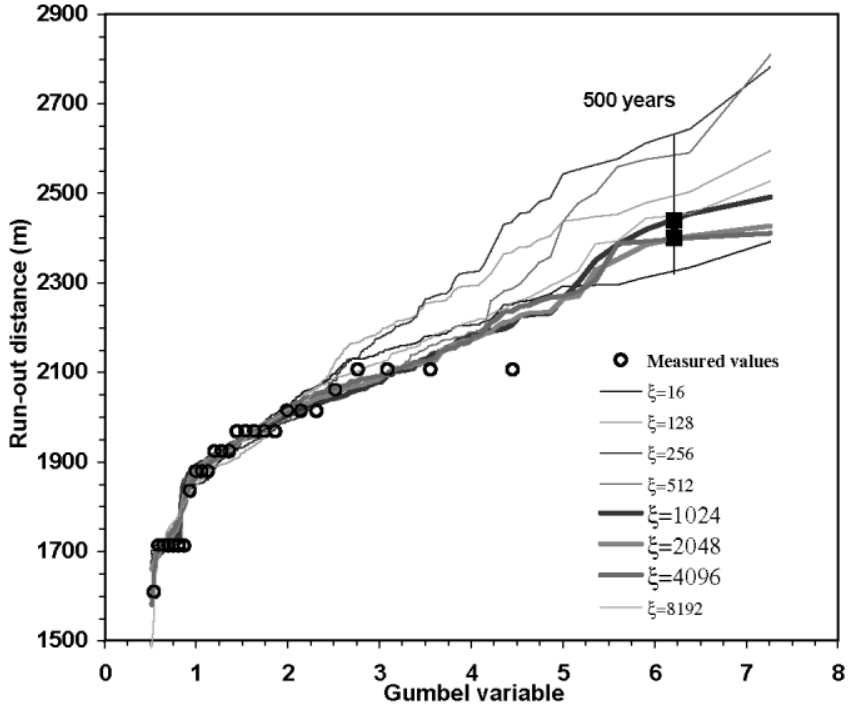

Fig. 12. Run-out distance statistical distributions with the $\mu$ samples, different fixed values of $\xi$ (in $\mathrm{m} \mathrm{s}^{-2}$ ), and the modified profile (Voellmy-like model).

To summarize, we conclude that:

(i) either the $(\mu \mid \xi)$ or the $(\xi \mid \mu)$ sample can be used;

(ii) the range of values for the fixed parameter is not completely free, and the choice of the variation range is important: it leads to an uncertainty of $50 \mathrm{~m}$ for the $(\mu \mid \xi)$ sample vs $90 \mathrm{~m}$ for the $(\xi \mid \mu)$ sample.

If we now compare these results with the 500 year runout distance obtained with the Coulomb-like model $(2269 \mathrm{~m})$, we see that the difference is approximately $50 \mathrm{~m}$, the Coulomb-like model providing a larger distance.

\section{COMPLEMENTARY ANALYSIS}

\section{Study of the modified profile with the Voellmy-like model}

The previous developments used the real profile whose final part was horizontal. In the Chamonix valley path, profiles of this kind are frequent, while in many other valleys in the French Alps, the slope variation is gentler. In order to test the conceptual approach in this case, we defined a modified profile (see Fig. 2) and made the same calculations as with the previous profile. The three first steps were the same, and the fourth step differed only in the path profile used in the propagation operator.

Only the results for the $(\mu \mid \xi)$ possibility are presented (see Fig. 12). The 500 year run-out distances obtained using the alternative possibility are reported in Table 3. Comparing Figures 10 and 12 shows that the bundles of curves are very similar below the key point of the horizontal terminal part of the profile and clearly differ above this point $(2100 \mathrm{~m})$. The range of results for the 500 year return-period run-out distances is very wide for the entire range of $\xi$ values $(310 \mathrm{~m})$, but very narrow for the limited range $(40 \mathrm{~m})$. This means that it is very important to use the ranges of practical values for the internal parameters rather than the entire ranges.

Table 3 shows that, once again, the run-out distances obtained using the $(\mu \mid \xi)$ values are larger than those obtained when the $(\xi \mid \mu)$ values were used. The difference 
Table 3. Comparison of the 500 year run-out distances obtained with the two models and for the two profiles

\begin{tabular}{|c|c|c|c|c|c|c|}
\hline & \multicolumn{3}{|c|}{ One-parameter model } & \multicolumn{3}{|c|}{ Two-parameter model } \\
\hline & \multicolumn{2}{|c|}{ Values of 500 years $X$ stop } & \multirow{2}{*}{$\begin{array}{l}\text { Differences between } \\
\text { (1) and (2) }\end{array}$} & \multicolumn{2}{|c|}{ Mean values of 500 years $X$ stop } & \multirow{2}{*}{$\begin{array}{l}\text { Differences between } \\
\quad(a) \text { and }(b)\end{array}$} \\
\hline & (1) Normal law & (2) Gumbel law & & (a) $\mu=0.23-0.46$ & (b) $\xi=900-5000 \mathrm{~m} \mathrm{~s}^{-2}$ & \\
\hline & 1000 years simulation & & & & & \\
\hline Real profile & 2384 & 2269 & 115 & 2206 & 2228 & -22 \\
\hline \multirow[t]{2}{*}{ Modified profile } & 2595 & 2382 & 213 & 2347 & 2420 & -73 \\
\hline & 20000 years simulatio & & & & & \\
\hline Real profile & 2360 & 2269 & 91 & 2205 & 2173 & 32 \\
\hline Modified profile & 2547 & 2380 & 167 & 2320 & 2394 & -74 \\
\hline
\end{tabular}

$(73 \mathrm{~m})$ is greater than for the flat profile $(22 \mathrm{~m})$. The Coulomb-like model gives a 500 year return-period runout distance of $2382 \mathrm{~m}$ situated between the two evaluations with the Voellmy-like model (2347 and $2420 \mathrm{~m}$ ). We can consider that in the conceptual approach, the use of each model is nearly equivalent when the Coulomb-like model is used with a Gumbel distribution for the $\mu$ values.

\section{Study of the friction parameter distribution with the Coulomb-like model}

In the conceptual approach, the variability of all the input variables and the friction parameter are reflected in the Monte Carlo simulations by their statistical distributions. Therefore, the choice of these distributions is of great importance, especially for the variables or the parameters that can reach large values (e.g. $H$ or $\xi$ in the Voellmy-like model) or come close to zero (e.g., $\mu$ in both models). This problem will not be studied completely, but we will provide a few indications in comparing the 500 year return periods obtained with the Coulomb-like model when the Gumbel and the normal distributions for the friction parameter $\mu$ are used. Figure 4 provides the non-exceeding probability of $\mu$ obtained using a normal distribution for $\mu$ instead of a Gumbel distribution. Although the two distributions provide similar results for the centre of the sample, they differ significantly for the extreme values, especially for the lower values of $\mu$. These differences entail substantial modifications (see Table 3): the results are much larger with the normal distribution than with the Gumbel distribution (115 $\mathrm{m}$ for the real profile and $211 \mathrm{~m}$ for the modified profile). These differences are greater than those observed when comparing the Coulomb-like and Voellmy-like models.

The role of the friction parameter distribution turns out to be a strategic one in the conceptual approach. This problem needs further investigation both on this path profile and on other profiles for which a large number of documented data are available.

\section{How large should the simulated samples be?}

In the preceding calculations we always retained the same samples of 450 random numbers, which gave either the $\mu$ sample for the Coulomb-like model or the $(\mu \mid \xi)$ (or the $(\xi \mid \mu))$ samples for the Voellmy-like model. The results obtained so far depend only on the model and the profile. Figures 10-12 show that the curves vary quite irregularly, implying that the confidence limits are large. In order to estimate the computation accuracy (i.e. taking the random uncertainty into account), the size of the simulation samples required to yield a confident estimation of the 500 year runout distance is sought. To that end, we will exemplify the method proposed for assessing the confidence interval by considering a particular case: in the computation, we will use the Voellmy-like model, the modified path profile (since it was shown that it amplifies data scattering) and the conditional probability of $\left(\mu \mid \xi=1024 \mathrm{~m} \mathrm{~s}^{-2}\right)$. As stated earlier, when $\xi=1024 \mathrm{~m} \mathrm{~s}^{-2}$, the influence of the starting position is sufficiently weak to be ignored in the simulations.

We therefore performed ten Monte Carlo simulations. For each of them, 450 events were simulated (as previously but each time with different random-number samples). Taken as a whole, these computations can be seen as either the simulation of the avalanche activity over a 10000 year interval or the reproducibility test of the avalanche activity over a 1000 year interval. As an example, the 500 year return period of the height $H$ computed with the 1000 year simulation is $7.5 \pm 1 \mathrm{~m}$. The run-out distance distributions are reported in Figure 13. Data scattering is substantial for individual samples and long periods of return. Similarly, the distribution related to the 10000 year interval is much smoother than individual distributions and provides an average trend around which the individual distributions vary. In this respect, the 10000 year distribution can be used to determine a quantile accurately while the individual distributions provide an idea of the possible variations around this value, that is, an estimate of the confidence limits. For instance, if we consider the 500 year quantile of the run-out distance, we infer from Figure 13 that the mean value is $2406 \mathrm{~m}$ and the confidence interval is approximately 2280 $2650 \mathrm{~m}$. The outcome is not very different if we take a longer interval of time (20 000 years): we obtain $2394 \mathrm{~m}$ instead of $2406 \mathrm{~m}$; the absolute difference between the two predictions is only $12 \mathrm{~m}$.

A practical rule emerges from this example: it is highly recommended to compute the desired quantile from a very large sample of simulations. Typically, if one is interested in determining the 500 year return-period avalanche, one should generate a sample corresponding to a continuous period of 10000 years (or more).

\section{Summary}

Table 3 sums up the results for the 500 year return-period run-out distances. The following conclusions can be drawn:

The predicted run-out distances obtained with the Coulomb-like model are larger with the normal distribution 


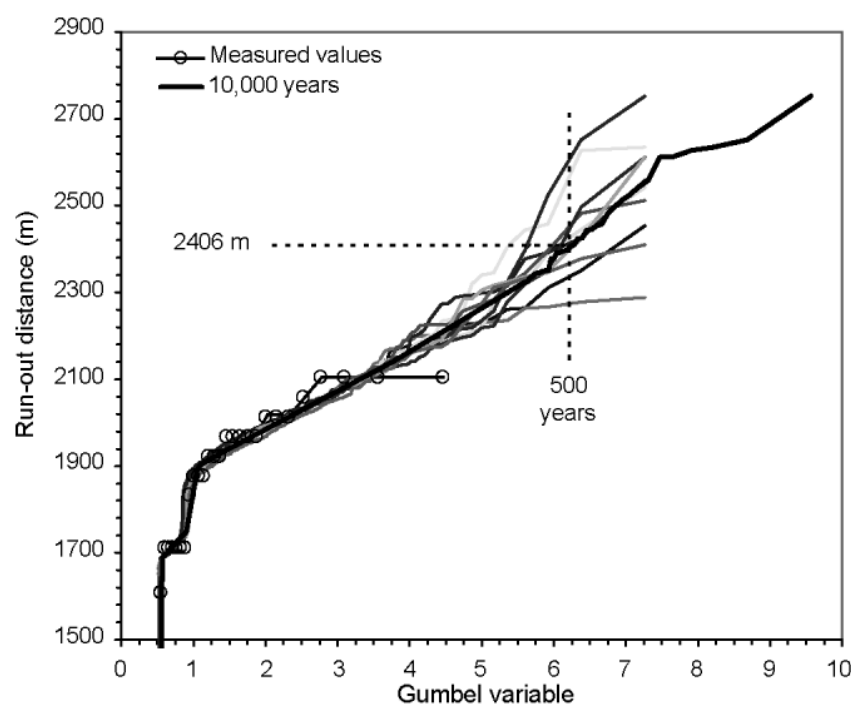

Fig. 13. Determination of the confidence limits of the run-out distances (Vollmy-like model) for the modified profile.

than with the Gumbel distribution. The difference ranges from $100 \mathrm{~m}$ with the real profile to $200 \mathrm{~m}$ with the modified profile.

The results given by the Voellmy-like model are different depending on the $(\mu \mid \xi)$ or the $(\xi \mid \mu)$ sample. The difference ranges from 20 to $70 \mathrm{~m}$, less than the difference introduced by the choice of the friction parameter distribution.

The results obtained with the two models are very similar except when using the normal distribution for the $\mu$ values with the Coulomb-like model.

\section{CONGLUSION}

In this paper we have developed a method to compute the run-out distances of long-return-period avalanches. Since this variable depends on both the path profile and avalanche dynamics, it is not licit to compute high-returnperiod run-out distances merely by extrapolating an empirical distribution fitted on the observed values.

Here we have suggested using a conceptual avalanchedynamics model and a four-step methodology. The first step involves choosing the input variables according to the available data and calculating the parameters of the avalanchedynamics model from the recorded historical events. The second step involves adjusting the statistical distributions of the input variables and of the model parameters. In the third step, Monte Carlo simulations are performed by making use of the previously determined statistical distributions. In this way, in the fourth step, we can generate a series of fictitious avalanches over a period of any duration. By taking a very long interval of time (typically 10000 years), it is possible to obtain an accurate estimate of the run-out distance of a very rare avalanche (500 year return period). This four-step methodology has been exemplified here using an avalanche path in the French Alps and two sliding-block models: the Coulomb-like and the Voellmy-like model.

We have shown that this methodology can be easily used with the Coulomb-like model, which needs only one frictional parameter. It is more complicated with the Voellmy- like model based on two frictional parameters. We have also demonstrated that the conceptual approach can be used with benefit in the avalanche field to compute a highreturn-period event. An important result is that the statistical distribution of the friction parameter plays a central role in the final results. Its choice is crucial, and further investigations are needed to clarify this point. Furthermore, no significant difference was found in the extrapolated quantile $x_{\text {stop }}$ when comparing the Coulomb-like and Voellmy-like models, when using the Gumbel law for the Coulomb-like model. Since the normal distribution seems to better fit the data for this model, it is necessary to study this point more thoroughly.

Finally, it seems that this methodology can be very useful for practical studies, but further research is required: applying it to many other paths and comparing the results with similar studies using the deterministic approach and studying the problem of the determination of the statistical distribution of the friction parameters using many different datasets. However, practitioners must be aware of just how important the field dataset is: in the French context, a large preliminary study would be required to assess the validity of the data. Extrapolating the statistical distributions of the internal parameters consistently with the data, the path profile and the model results must also be investigated. These two requirements may be difficult to meet, but this work is well worth the effort, since this is how long-returnperiod run-out distances can be determined scientifically and consistently. At the very least, the comparison with the results given by empirical methods will always be useful.

\section{ACKNOWLEDGEMENTS}

This study was supported by Cemagref, and funding was provided by the European programme CADZIE (EVG1CT-1999-0009). The authors are grateful to M. Naaim for providing the numerical code of the Voellmy-like model.

\section{REFERENCES}

Ancey, C. 2001. Snow avalanches. In Balmforth, N. J. and A. Provenzalle, eds. Geomorphological fluid mechanics. Berlin, Springer-Verlag, 319-338. (Lecture Notes in Physics 582.)

Ancey, C., M. Meunier and D. Richard. 2003. The inverse problem in avalanche-dynamics model. Water Resour. Res., 39(4), Suppl. 1099. (1029/ 2002WR001749.)

Bakkehøi, S., U. Domaas and K. Lied. 1983. Calculation of snow avalanche runout distance. Ann. Glaciol., 4, 24-29.

Barbolini, M. and F. Savi. 2001. Estimate of uncertainties in avalanche hazard mapping. Ann. Glaciol., 32, 299-305.

Barbolini, M., U. Gruber, C. J. Keylock, M. Naaim and F. Savi. 2000. Application of statistical and hydraulic-continuum dense-snow avalanche models to 5 real European sites. Cold Reg. Sci. Technol., 31 (2), 133-149.

Bates, B. C. and E. P. Campbell. 2001. A Markov chain Monte Carlo scheme for parameter estimation in conceptual rainfall-runoff modelling. Water Resour. Res., 37(4), 937-947.

Betson, R. P. and C.V. Ardis. 1989. Implications for modelling surfacewater hydrology. In Kirkby, M. J., ed. Hillslope hydrology. New York, John Wiley and Sons, 295-323.

Beven, K. 1989. Changing ideas in hydrology — the case of physicallybased models. F. Hydrol., 105(1-2), 157-172.

Buser, O. and H. Frutiger. 1980. Observed maximum run-out distance of snow avalanches and the determination of the friction coefficients $\mu$ and $\xi$. F. Glaciol., 26(94), 121-130

Dent, J. D. and T. E. Lang. 1980. Modeling of snow flow. F. Glaciol., 26(94), 131-140.

Fujisawa, K., R. Tsunaki and I. Kamiishi. 1993. Estimating snow avalanche runout distances from topographic data. Ann. Glaciol., 18, 239-244. 
Ghali, A. 1995. Méthodes statistiques pour la détermination de la distance d'arrêt des avalanches. La Houille Blanche, 100-104.

Harbitz, C.B. 1999. Snow avalanche modelling, mapping and warning in Europe. Deliverable No. 4. A survey of computational models for snow avalanche motion. Oslo, Norwegian Geotechnical Institute. (NGI Technical Report 581220-1.)

Keylock, C.J., D. M. McClung and M. M. Magnússon. 1999. Avalanche risk mapping by simulation. F. Glaciol., 45(150), 303-314.

Lied, K. and S. Bakkehøi. 1980. Empirical calculations of snow-avalanche run-out distance based on topographic parameters. f. Glaciol., 26(94), 165-177.

McClung, D. M. 2000. Extreme avalanche run-out in space and time. Can. Geotech. 7., 37, 161-170.

McClung, D. M. 2001. Extreme avalanche run-out: a comparison of empirical models. Can. Geotech. F., 38, 1254-1265.

McClung, D. M. and K. Lied. 1987. Statistical and geometrical definition of snow avalanche runout. Cold Reg. Sci. Technol., 13(2), 107-119.

Mears, A. I. 1992. Snow-avalanche hazard analysis for land-use planning and engineering. Colorado Geol. Surv. Bull., 49.

Meunier, M. 1991. Eléments d'hydraulique torrentielle. Antony, Cemagref Editions. (Séries Etudes Montagnes, 1.)

Meunier, M., C. Ancey and M. Naaim. 2001. Mise au point d'une méthode de prédétermination statistique des cotes d'arrêt d'avalanches. La Houille Blanche, 92-98.

O'Connell, P. E. and E. Todini. 1996. Modelling of rainfall, flow and mass transport in hydrological systems: an overview. F. Hydrol., 175(1-4), 3-16.

Salm, B. 1993. Flow, flow transition and runout distances of flowing avalanches. Ann. Glaciol., 18, 221-226.

Salm, B., A. Burkard and H. Gubler. 1990. Berechnung von Fliesslawinen: eine Anleitung für Praktiker mit Beispielen. Eidg. Inst. Schnee- und Lawinenforsch. Mitt. 47.

Schaerer, P. A. 1975. Friction coefficients and speed of flowing avalanches. International Association of Hydrological Sciences Publication 114 (Symposium at Grindelwald 1974 - Snow Mechanics), 425-432.

Vanderkwaak, J. E. and K. Loague. 2001. Hydrological response simulations for the R-5 catchment with a comprehensive physics-based model. Water Resour. Res., 37(4), 999-1013.

\section{APPENDIX}

The mean flow depth $H$ can be estimated from the recorded avalanche volumes using assumptions similar to the empirical arguments used for debris flows, which were roughly validated by Russian experiments (Meunier, 1991, p. 208):

(i) For natural flows, a relationship exists between height and width, for which a power-law function can be used. For water discharge, the exponent is zero for the rectangular section, equal to 1 for the triangular section, and less than 1 for convex sections. Here we assume that the width of the avalanche flow $W$ is a power function of the avalanche height: $W \propto H^{0.7}$. This argument is more doubtful for an open slope avalanche, depending on the rheological behaviour of avalanche snow.

(ii) Similarly, the length $L$ of the avalanche flow is a power function of the avalanche height, but with a lower exponent: $L \propto H^{0.3}$. Thus, we obtain a crude relationship between the avalanche volume and its height: $V \propto H^{2}$. This expression holds on average but not necessarily for every event.

(iii) We assume that the avalanche flow depth ranges from 2.5 to $8 \mathrm{~m}$.

(iv) The avalanche volume is assumed to be constant during the avalanche run and deposition (snow entrainment and compaction are ignored).

Combining all these relationships and assumptions, we finally obtain the following result linking $H$ and $V$ : $H=2.5+\left(5 \times 10^{-3}\right) V^{1 / 2}$. 